\title{
Machine Learning for Novel Thermal-Materials Discovery: Early Successes, Opportunities, and Challenges
}

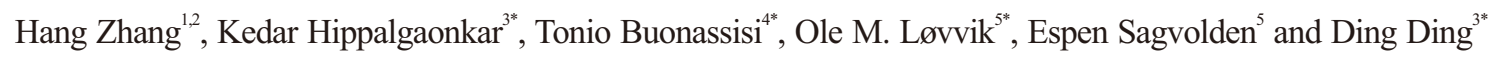

High-throughput computational and experimental design of materials has becoming an increasingly important field in material science overall. However, this area of research has largely been only been emerging in thermal sciences, in part due to the computational and experimental challenges faced in obtaining thermal properties of materials. In this paper, we provide a current overview of some of the recent work and highlight the challenges and opportunities that are ahead of us in this field. In particular, we focus on the use of machine learning and highthroughput methods for screening of thermal conductivity for compounds, composite and alloys and interface conductance. These methods have brought about a feedback mechanism for understanding new correlations and identifying new descriptors, speeding up the discovery of novel thermal materials.

Received 31 December 2018

DOI: $10.30919 /$ esee8c209

\section{Introduction}

As humanity's energy use increases, so too do demands on materials' thermal and thermal-transport properties. For example, materials with thermal conductivities $(\kappa)$ below $1 \mathrm{~W} / \mathrm{mK}$ are needed for thermal insulation $^{1}$, and above $10,000 \mathrm{~W} / \mathrm{mK}$ for heat management on nextgeneration consumer electronics and energy-generation technologies. ${ }^{2}$ To gain market acceptance, new materials must also satisfy other application-specific constraints, including electrical conductivity, cost, density, manufacturability, mechanical properties, durability, chemical compatibility, and environmental impact. ${ }^{3}$ From a scientific point of view, thermal and thermal-transport properties are governed by a handful of underlying materials properties factoring into the Boltzmann Transport Equation (BTE) that depend on structure and atomic constitution. ${ }^{4}$ In essence, the materials-innovation challenge in thermal sciences is more complex than multi-parameter optimization, as it involves not just the search for new materials, but the search for new physics.

Increasingly, materials science researchers are applying combinations of emergent machine learning (ML), high-performance computing (HPC), and automation tools to accelerate the rate of novel materials discovery and development ${ }^{5}$. This transformation in how we

${ }^{1}$ Institute of Engineering Thermophysics, Chinese Academy of Sciences, Beijing 100190, PR China

${ }^{2}$ University of Chinese Academy of Sciences, Beijing 100049, PR China

${ }^{3}$ Institute of Materials Research and Engineering, A*STAR Research Entities, 138634 Singapore

${ }^{4}$ Massachusetts Institute of Technology, Cambridge 02139, MA, USA

${ }^{5}$ Department of Physics, University of Oslo, 0316 Oslo, Norway

*E-mail: kedarh@imre.a-star.edu.sg(K.H.); buonassisi@mac.com

(T.B.); olemartin.lovvik@sintef.no(O.L.); ding_ding@SIMTech.a-

staredu.sg (D.D.) perform R\&D reflects the community's desires for faster cycles of learning, deeper physical insights, greater sophistication in how we design, synthesize, and optimize materials, and a recognition that we must push physical limits if we are to make meaningful advances in this field in market- and societally-relevant timeframes. This review focuses on the thermal sciences, and is divided into two parts. First, we assess the state of the art in applying machine-learning methods to accelerate materials development. Second, we review current challenges attracting researchers' attention, as well as underserved areas in machine-learning methods for thermal sciences. In the outlook section, we describe future work standing between our present reality and a future vision of fullyautomated, self-driving laboratories that enable accelerated materials discovery and development.

As shown in Fig. 1, early successes focus on high-accuracy yet computationally expensive HPC to calculate underlying materials properties governing thermal transport. These parameters, which include phonon density of states ${ }^{6}$, thermal conductivity ${ }^{4}$, Debye temperature? and the elastic properties ${ }^{8-12}$, can now be routinely computed using variants of density functional theory (DFT) $)^{13,14}$ with reasonable accuracy ${ }^{4}$, albeit at a rate of a few dozens of compounds per year. To increase throughput and enable screening on the scale of hundreds of thousands of compounds, heuristic models and numerical approximations have been developed ${ }^{15}$, albeit with accuracies typically in the range of $20 \%$, and with limited accuracy beyond interpolation. Additionally, new parameters of merit, for example the large splitting of acoustic and optical phonon branches exemplified in boron arsenide ${ }^{16}$, are being reported at a rate of a handful per decade. To experimentally validate these predictions, consensus has emerged surrounding best practices for thermal property and transport characterization ${ }^{17}$, which eliminate most experimental artifacts and establish community-wide benchmarking ${ }^{18}$.

Looking ahead, one important challenge is to develop faster and more accurate predictors of materials descriptors, toward enabling materials searches including millions of compounds. Machine learning has proven useful in this and related domains, to accelerate, augment, 
and even leapfrog DFT, revealing difficult-to-calculate parameter $\kappa .^{19,20}$ (Fig. 1). Ultimately, extending these predictive tools beyond interpolation may enable new physics-based descriptors to be discovered. High-throughput synthesis tools hold promise to solve the multiparameter optimization challenge intrinsic to the thermal sciences and related energy fields, as well as to provide valuable feedback to refine theoretical models. Machine-learning methods will be challenged by the unique topology of the thermal sciences, including the high degree of correlation between parameters influencing the BTE, complexity (e.g., microstructures and composition) across multiple length scales, and sparse - but extremely rich — data sets. Data challenges include transferring learnings across different platforms, and integrating disparate data repositories. Physics-based challenges include exploring wave effects of phonons, exceeding the amorphous (CahillPohl) limit $^{21}$ on the low end of thermal conductivity, and extending phonon lifetimes on the high end.

The thermal properties of materials are very important for understanding thermodynamic stability of structural phases and their suitability for a variety of applications. High thermal conductivity materials are essential for efficient heat removal while low thermal conductivity materials can give rise to the next generation of thermoelectric materials and thermal barriers. Within computational abilities and experimental fabrication and testing, our community has successfully predicted ${ }^{16}$, synthesized and experimentally measured the highest thermal conductivity material know to date ${ }^{22-24}$. At the same time, computational predictions and experimental verification have also led to discovery of a number of low thermal conductivity materials ${ }^{25-29}$. However, there are not many systematic studies of classes of materials to guide us into understanding what material attributes contribute towards thermal conductivity.

To do so, we will require a high throughput platform whereby computational screening and experimental testing can be conducted on a large number of samples within reasonable computational and experimental resources ${ }^{5}$. High-throughput (HT) computational screening is a rapidly expanding area of materials research ${ }^{30}$. Increasing availability of computational resources have resulted in large databases, and has generated, for example, the AFLOWLIB.org consortium ${ }^{31}$ the Materials Project database ${ }^{30,32,33}$, Citrination ${ }^{34}$, among others ${ }^{35}$. These databases and the application of HT has recently led to new insights and novel compounds in different fields ${ }^{35-40}$. However, despite the importance of thermal transport properties for many crucial technologies, there are to date only a few HT investigations into lattice thermal conductivity ${ }^{15,19,20,41,42}$. One main issue is that the determination of the thermal conductivity of materials is computationally demanding as it requires calculation of multiple-phonon scattering processes. The third-order anharmonic IFCs (inter-atomic force constants) required in order to account for three-phonon scattering processes ${ }^{4}$ with standard ways such as density functional theory (DFT) and density functional perturbation theory (DFPT) ${ }^{43}$ are generally computationally expensive. This can either be based on the frozen-phonon approach ${ }^{44}$ or the temperature-dependent effective potential (TDEP) method ${ }^{45}$, the latter based on first-principles molecular dynamics calculations at explicit temperatures, with the option to generate a canonical ensemble of supercell configurations using Monte Carlo sampling. An alternative approach to calculating thermal conductivity is based on the GreenKubo formulation which employs molecular dynamics simulations to calculate heat fluxes upon thermal equilibrium ${ }^{46,47}$. This technique accounts for high-order scattering processes, but semi empirical

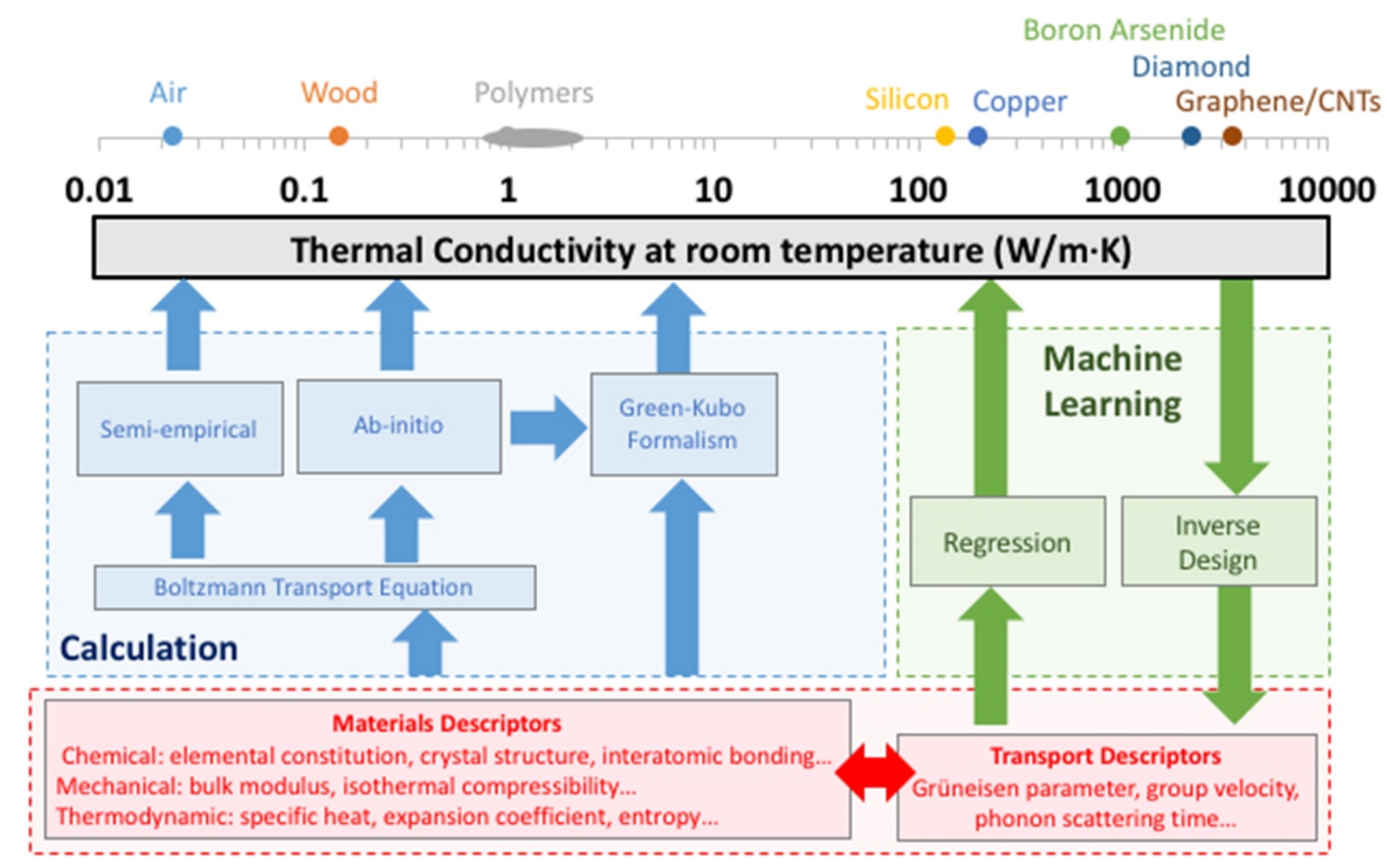

Fig. 1 Thermal Conductivity can range on a few orders of magnitude. Obtaining accurate values of thermal conductivity and understanding the physical reason for the thermal conductivity is always a challenge as calculations are computationally expensive and is only a one-way approach of tweak and observe. With Machine Learning, the cycle of material discovery is now complete where we can correlate thermal conductivity with descriptors, providing the feedback to speed up materials innovation and discovery. 
potentials used in these calculations can lead to errors on the order of $50 \%{ }^{48}$.

A variety of simple methods have been devised to evaluate the thermal properties of materials at reduced computational cost. Early approximate implementations to compute the lattice thermal conductivity were based on semi empirical models to solve the BTE, in which some parameters are obtained from fitting to experimental data ${ }^{49}$, ${ }^{50}$. This reduces the predictive power of the calculations. The methods described above are unsuitable for rapid generation and screening of large databases of materials properties in order to identify trends and simple descriptors for thermal properties ${ }^{5}$.

\section{Early successes - theoretical modeling of thermal properties}

\section{a. bulk stoichiometric compounds}

Despite the computational cost, there has been a few attempts to generate HT data for predicting thermal properties. The first large scale HT attempt was done by Carrete et al. ${ }^{19}$ The work concentrated on halfHeusler (HH) compounds where unstable and zero-gapped compounds were screened by DFT. Then, full DFT calculations for $\mathrm{k}$ for a smaller subset of such compounds were carried out as a training set upon which random forest regression is used to build a classification model for the descriptors. IFCs were also predicted from random forest algorithm upon which good agreement between exact $\mathrm{k}$ and predicted $\mathrm{k}$ have been obtained (Fig. 2a). It is found that compounds are most likely to have low thermal conductivity if the average atomic radius of the atoms in structural positions A and B is large. HT screening based on Bayesian optimization was applied to a larger set of materials by Seko et al. (Fig. $2 c{ }^{20}$. Two descriptors, namely density and crystal volume per atom, were used to provide a score upon which material databases can be screened in a HT fashion.

Despite these speed ups with ML methods, a small set of training data is still required upon which full calculations are necessary. There exist parallel efforts to develop computationally less expensive ways of calculating k. Original models such as semi-empirical methods by Allen ${ }^{49}$ and Callaway ${ }^{50}$ usually requires fitting parameters from experimental data. A recent attempt by Miller et al. (Fig. 3a) ${ }^{15}$ uses DFT data to fit to a semi empirical model based on Debye Callaway model. However, most HT approaches still rely on DFT to some extent Recently, a much less computationally expensive approach (Fig. 3b) ${ }^{42}$ used the "GIBBS" quasi-harmonic Debye model ${ }^{7}$ to enable a fast HT method for computing $\mathrm{k}$ for a large class of materials. This approach only requires electronic DFT calculations and not calculations of the IFCs. At the same time, principal component analysis has been used to extrapolate IFCs at finite temperatures from a few sets of full IFC calculations to predict the thermal stability and $\mathrm{k}$ at finite temperatures ${ }^{41}$. Qin and $\mathrm{Hu}^{51}$ described a way based on the analysis of the harmonic (second order) IFCs to accelerate the evaluation process of obtaining accurate $\mathrm{k}$ by solving the cutoff distance problem. More recently efforts have been devoted to evaluate the phonon band structures and evaluation of thermodynamic properties for a large number of compounds ${ }^{52}$. For instance, Atsushi Togo's phonon database (http://phonondb.mtl.kyoto-u.ac.jp) with full phonon band structures and derived quantities for 1521 semiconducting inorganic crystal were recently reported (Fig. 3c) ${ }^{6}$. With further development in HT methods for IFCs, we foresee that truely HT computational screening of $\mathrm{k}$ with DFT is possible in the near future.

\section{b. Aperiodic composites and porous materials}

Unlike periodic compounds, composite materials and porous media have wide engineering applications but their effective thermal conductivity is a problem that first principle atomistic methods typically cannot tackle. To predict the effective thermal conductivities of composite materials, existing methods such as effective medium theory (EMT) ${ }^{53}$, heat diffusion equation ${ }^{54}$, and Boltzmann transport equation (BTE) ${ }^{55}$ have been used. The EMT provides analytical models that can estimate the effective thermal conductivities of the composite materials but its accuracy is limited as it does not account for the effect of the distribution of constituent materials. In order to take in to account the details of materials distribution in a composite, direct solutions of heat diffusion equation will be necessary. Many numerical methods such as finite volume method (FVM) ${ }^{56}$, the finite element method (FEM) ${ }^{54}$ and the lattice Boltzmann method (LBM) ${ }^{57}$ have also been developed. All these approaches are based on solving partial differential equations (PDE) which are computationally costly. Recently, Zhang et al. ${ }^{58}$ designed a genetic algorithm to optimize the configuration of silicon
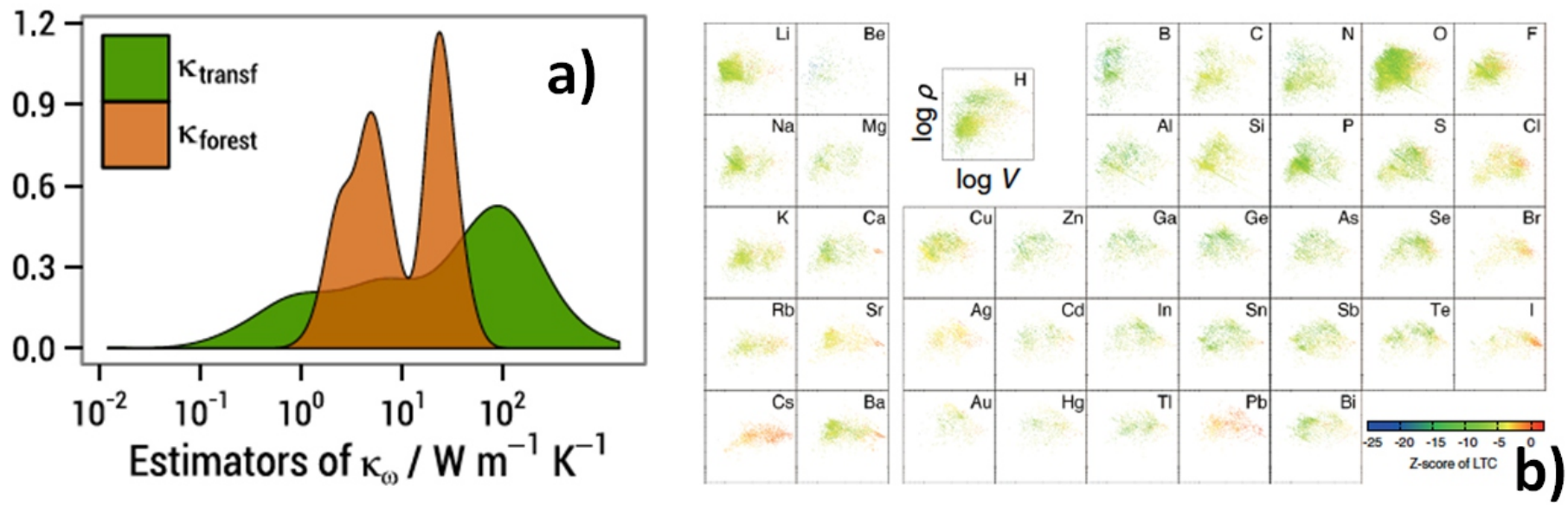

Fig. 2 (a) Frequency densities of the estimators of thermal conductivity at $300 \mathrm{~K}$. $\kappa_{\text {trans }}$ is Approximated $\kappa_{\omega}$ with anharmonic force constants from Mg $\mathrm{Si}$ and $\kappa_{\text {forst }}$ is $\mathrm{k}$ prediction obtained random-forest regression. (b) Dependence of the Z score on constituent elements for compounds in the MPD library. The magnitude of the $\mathrm{Z}$ score is shown by colors along with volume, $\mathrm{V}$, and density, $\rho$, for each element. 
germanium composites under multi-parameters for the best thermal conductivities (Fig. 4). Possessing the advantages of low cost on both coding and computational expense, this approach can be feasibly grafted for solving optimization problems on thermal properties of other composites.

In another work by Wei et al. ${ }^{59}$, machine learning methods including Support Vector Regression (SVR), Gaussian Process Regression (GPR) and Convoluted Neural Networks (CNN) were used to study the heat transfer in composite materials and porous media. SVR and GPR are non-linear regression methods that provides thermal conductivity classification with descriptors while $\mathrm{CNN}$ can handle much more descriptors with the given data. The difference is that EMT is based on physical understanding of the system while machine learning is based solely on data analysis. Wei et al. created a database using the quartet structure generation set (QSGS) to generate composite material structure and applying LBM to calculate the effective thermal conductivity. Then, this database is used to train and test the different machine learning methods. All three methods have shown good predictions as a fraction of the computational cost. CNN is the most comprehensive in being able to handle large datasets but it is only accurate if more expensive to training with larger datasets is given. This is in sharp contrast compared to SVR and GPR where smaller number of descriptors can provide reasonable correlation.

\section{c. Alloys - using High Entropy Alloys as a test case}

A particularly interesting group of materials exhibiting exotic properties and ultra-low thermal conductivity is formed when mixing several elements on an atomic level with random occupation of each lattice site, i.e. solid solution. However, it is challenging to describe such materials from first principles. Since there is perfect disorder in which atom occupies which position, there is no longer a well-defined translational symmetry. A particularly interesting test-case of such multi-principal element alloys are high-entropy alloys (HEAs) when they are based on at least five different elements and constitute a single phase with solid solution ${ }^{60}$. Alloying is a well-known technique to reduce the thermal conductivity, and the HEA concept has therefore been used to minimize thermal conductivity in several studies, often with an emphasis to develop novel thermoelectric materials ${ }^{61}$.

Several methods have been employed to describe HEAs on the electronic scale ${ }^{62}$, including the virtual crystal approximation (VCA) ${ }^{10}$, coherent potential approximation (CPA) ${ }^{63}$, special quasi-random structures (SQS) ${ }^{12,6468}$, and molecular dynamics (MD) based methods ${ }^{69}$

${ }^{71}$. Only a few studies have assessed the thermal conductivity of HEAs, using semi-empirical MD ${ }^{72,73}$. As mentioned above, the error involved in such methods may be quite high. This is particularly so for complex compounds with a large number of pair- and higher-order potentials. The studies only describe qualitative features of heat transport in generalized HEAs and are thus not suited for HT investigations. The VCA method has been used to predict thermal conductivity of solid solution alloys. It is based on first principles and requires relatively expensive calculations of interatomic force constants within a phonon scheme. Nevertheless, interpolation of force constants makes this method rather efficient, and has been shown to reproduce experimental data relatively well in e.g. the entire ternary phase diagram of the solid solution (Ti,Zr,Hf)NiSn system (Fig. 5a) ${ }^{74}$. In order to obtain reliable and predictive results, however, the most applicable of the above methods to computing thermal transport appears to be the SQS construction. In this method, the atomic positions of a supercell of finite size are designed to ideally mimic the nuclear pair-correlation function of the solid solution. The larger the cell, the better the pair-correlation function can be approximated. The technique gives excellent results; 128-atom supercells were recently found to reproduce very well the experimental thermal conductivity of the random alloy $\operatorname{In}_{1-x} \mathrm{Ga}_{x}$ As using SQS with a Green's function approach ${ }^{75}$. It was shown in the same study that disorder of the interatomic force constants was necessary to obtain good correspondence with experiment, rendering the VCA approach significantly less accurate (Fig. 5b).

So far, no studies in the literature have to our knowledge predicted the calculated thermal conductivity of a HEA based on SQS at the DFT level. It can be anticipated that such studies will be available quite soon, and that they will form the basis of HT studies aiming at developing HEAs with extremely low thermal conductivity. Since these materials often come with other extraordinary properties, it can be expected that accelerated discoveries of novel HEAs will lead to materials with

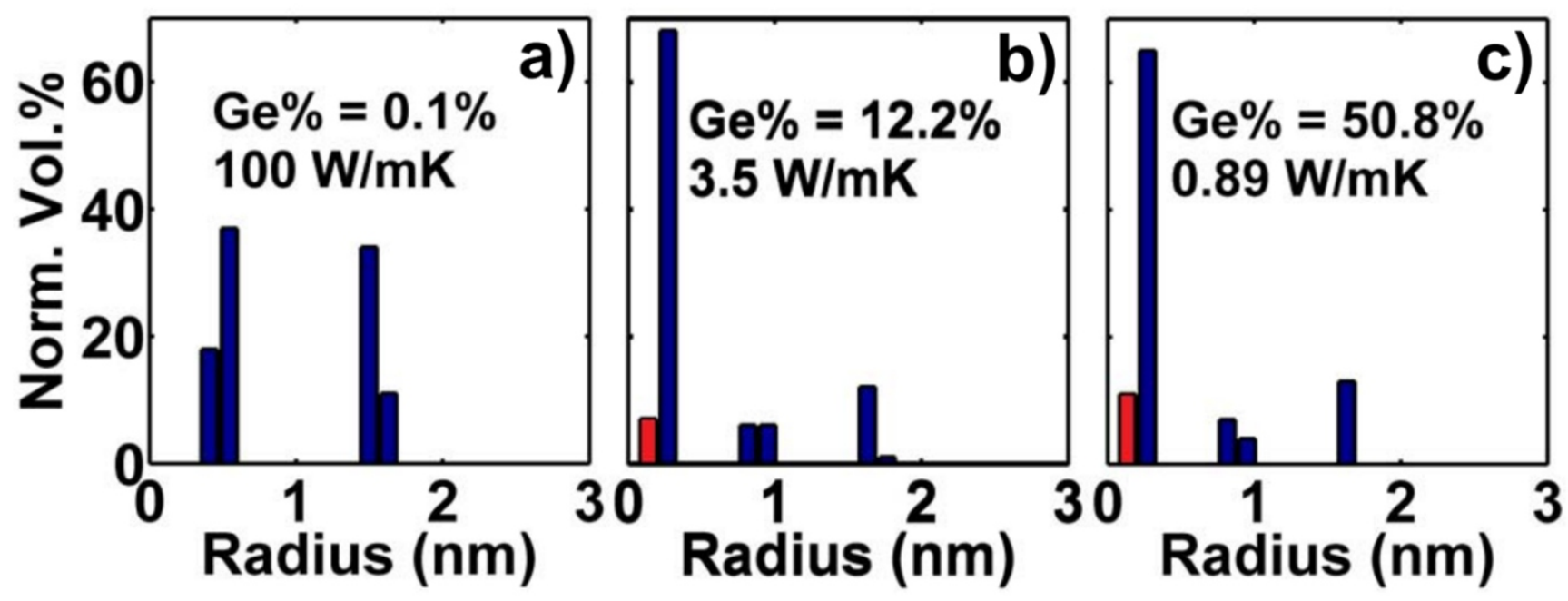

Fig. 4 Optimal size distribution of Ge nanoparticles for lowest thermal conductivity of $\mathrm{Si}_{\mathrm{x}} \mathrm{Ge}_{1-\mathrm{x}}$ composites. Heights of histogram bars indicate fraction of Ge allocated to each nanoparticle size. The best distribution introduces additional non-adjacent peaks as more Ge is added. Red histogram bars indicate point defects. 
unique combinations of thermal and other properties.

\section{d. Interface thermal conductance - beyond the Acoustic and Diffuse Mismatch models}

Ever since Kapitza ${ }^{76}$ discovered that a non-continuous temperature drop at the interface between helium and a solid, thermal boundary resistance has becomea central problem to thermal material design. The thermal interface resistance between two materials is the ratio of the temperaturte discounity at the interface to the power per unit area flowing across the interface. The Acoustic Mismatch Model (AMM) and the Diffuse Mismatch Model (DMM) ${ }^{77,78}$ provide the upper and lower bounds for such an estimate, assuming no scatter for the former and complete diffuse scattering for the latter. Such models have been accurate for solid-solid interface and provided significant insight into the mechanics of phonon transport at the interface - the group velocity of the phonons and the overlap of the phonon density of states being two key physical descriptors that are responsible for how heat flows at an interface. However, it is not accurate for describing interfaces with real defects and roughness ${ }^{78}$. A significant advancement was made due to full calculations of the phonon density of states and integrating over this in the Boltzmann transport simulations or Green-Kubo (numerical or analytical integration techniques) ${ }^{79}$ allows for the description of frequency dependent interfacial conductance. However, such methods can lead to a loss of accuracy by discounting effects such as intermixing at the interfaces, roughness effects and electron-phonon coupling, and not properly accounting for the finite size effects of the simulation domain ${ }^{80}$. While lots of work have shown the limited validity of AMM and DMM ${ }^{81,82}$ and new models have been proposed ${ }^{83,84}$, ML methods can certainly bridge this gap in knowledge, by identifying the key physical attributes necessary to accurately predict the thermal interface properties.

Two recent works have utilized ML methods to understand interfacial thermal resistance. The work by Juni Shiomi's group ${ }^{85}$ explored the use of Bayesian optimization for computing interfacial thermal conductance (ITC) of super lattice structures consisting of $\mathrm{Si}$ and Ge (Fig. 6a). ITC can be minimized or maximized by combining Atomistic Green's Function (AGF) with Bayesian Optimization just by calculating only a few percent of all possible structures, leading to considerable saving in computational resources. It is also found that aperiodic structures can minimize ITC more due to a lack of phonon coherence. The recent work by Yibin Xu's group ${ }^{86}$ utilizes online and published data with the acoustic mismatch model and diffuse mismatch model. Using regression methods including SVR, GVR, accurate prediction of interfacial thermal resistance has been achieved. It is found that AMM and DMM are not good descriptors while melting point and heat capacity are good descriptors (Fig. 6b). At the same time, ML and NN have recently been employed to study interfacial thermal resistance between graphene and hexagonal boron nitride ${ }^{87}$.

\section{Experiments}

While HT predictions of $\mathrm{k}$ through ML have been explored, experimental efforts to realize this realm have been lacking. In principle, techniques such as time domain thermoreflectance (TDTR) are perfectly suited for fast, non-destructive testing of thermal properties $^{88}$. Implemented with sophisticated modeling based on the approaches described above, these high throughput experimental tools can ascertain the thermal conductivity, specific heat (extracted from the thermal diffusivity), interface thermal conductance as well as anisotropic thermal properties. Going beyond, because of the depth of knowledge developed by the community in this regard, the intrinsic transport descriptors such as the dominant phonon wavelengths participating in thermal transport, scattering times, mean free path accumulation functions and more have also been gleaned out. Combined with highthroughput materials synthesis techniques ${ }^{89,90}$ both in the solid solution and combinatorial thin film form, there is a huge opportunity here where such HT experimental techniques can provide high-fidelity data that will not only provide insight on what dictates thermal transport, but also provide a database that can serve as a test-set for ML algorithms. Similar approaches have already proven successful for small molecules and proteins ${ }^{91,92}$ and the need-of-the-hour is to leverage build upon fast
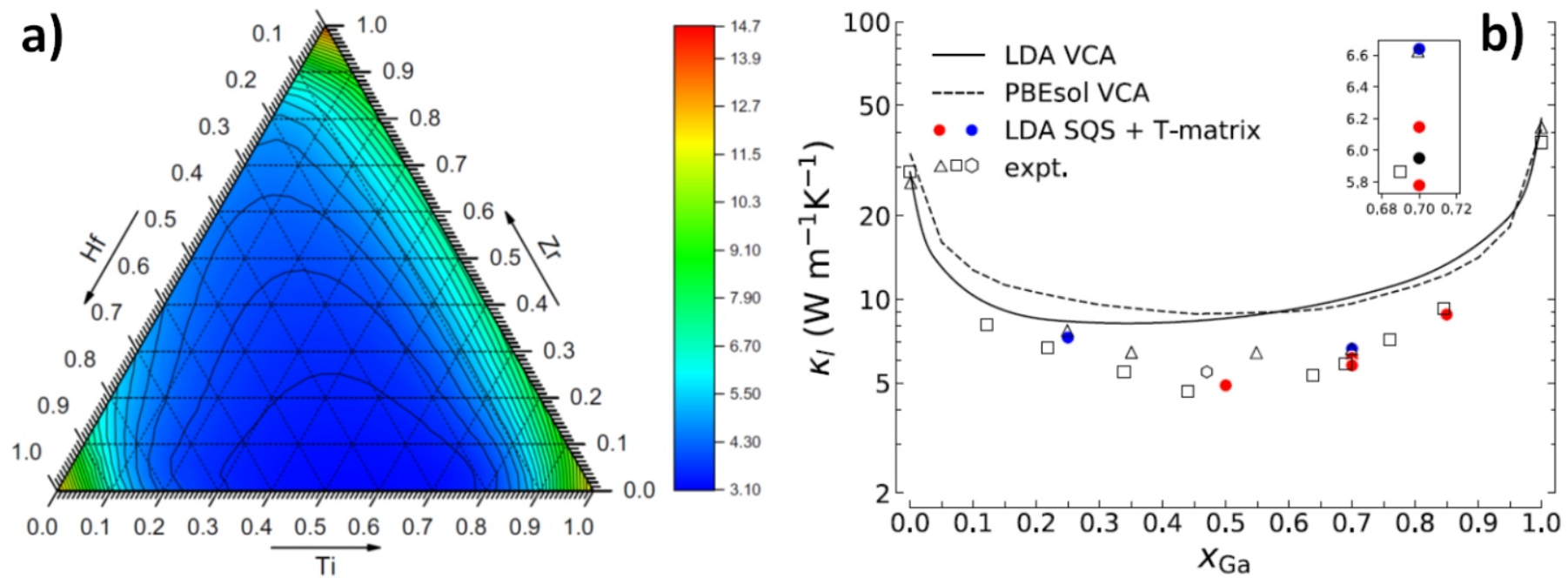

Fig. 5 The phonon part of the thermal conductivity calculated with DFT employing semiclassical Boltzmann transport equations. (a) $\mathrm{Ti}_{x} \mathrm{Zr}_{\mathrm{y}} \mathrm{Hf}_{1-x-y} \mathrm{NiSn}_{\mathrm{St}}$ $300 \mathrm{~K}$ using the virtual crystal approximation (VCA). The bottom right corner corresponds to TiNiSn, the top to ZrNiSn, and the bottom left to HfNiSn. (b) $\mathrm{In}_{1-x} \mathrm{Ga}_{x} \mathrm{As}$ at $300 \mathrm{~K}$ using VCA (solid line based on the local density approximation (LDA) and dashed line based on the PBEsol generalized gradient approximation) and the special quasi-random structure (SQS) technique (red and blue dots based on LDA with 128- and 250-atom supercells, black dot based on PBEsol). Experimental results are shown with the open symbols. Picture taken from Refs. ${ }^{74}$. . Printed with permission from ... 


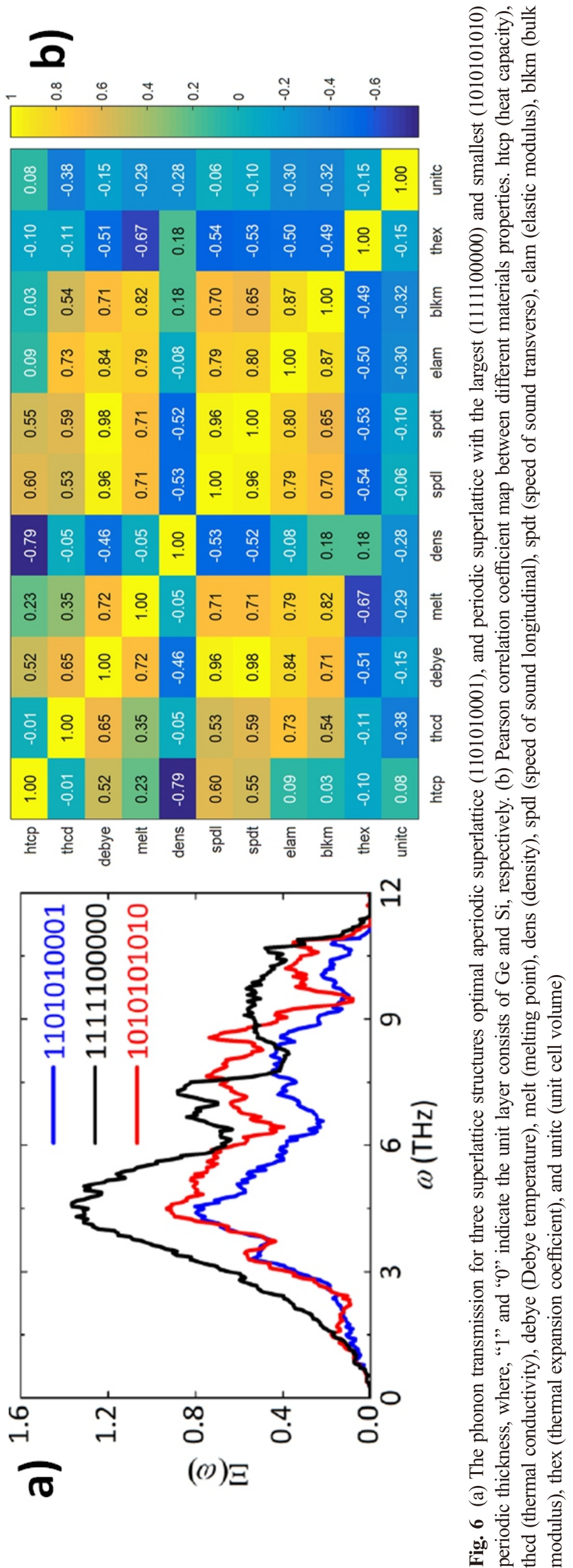

experimental characterization tools to create a thermal material property library. For example, measuring the in-plane and cross-plane thermal conductivities of a material grown by combinatorial synthesis could go a long way towards designing new thermal materials. The work by David Cahill's Group leverages upon time domain thermoreflectance to measure $\mathrm{k}$ experimentally for nickel solid solutions ${ }^{8893}$. Such HT techniques have already been being developed for chemistry ${ }^{94}$, solar cell materials $^{90}$ and batteries ${ }^{95}$.

Sophisticated tools such as Bayesian inference is able to use a forward model (similar to the Boltzmann Transport Equation) in order to extract hidden materials and transport properties simultaneously. Here, by looking at a system-level model in a PV cell, the authors were able to extract the bulk and interface properties that are limiting it's performance ${ }^{38}$. Similar new hardware approaches can also be developed for thermoelectrics. One can envision utilizing such ML algorithms coupled with advanced statistical analysis to provide experimental tools (for example extending the theoretical models used with TDTR) to provide fast screening for measurement of thermal properties of a large class of compounds. Large scale synthesis of bulk and thin film materials with varying stoichiometry and physical properties is an outstanding challenge that will impact not just the thermal community but other fields of materials science as well. In addition, a clear and present opportunity is to leverage upon existing theoretical databases and integrating them into a common language that is widely available to the researchers working in this area. This will enable an artful application of ML to sparse, but high-quality datasets (experimentally generated, but amplified by theoretical calculations). The general approach here is to provide high-throughput experimental data embellished by fast theoretical predictions, while high-fidelity careful measurements can subsequently be performed after this initial screening process. Since little work has been performed in this area, there is a vast space for discovery of new materials with novel thermal properties and exhibiting new physics; for example, moving beyond the classical size effects and scattering of phonons as particles, towards wave and coherent effects $^{96}$. Such high-throughput synthesis and thermal characterization will also enable the development of holistic understanding of thermal properties on material classes, bonding, alloys, microstructures, defects (both from understanding and moving towards engineering).

\section{Conclusions and Outlook}

Thanks to the establishment of materials database frameworks for and the rapid development on both computational hardware and algorithms for machine learning, successful pioneer works have emerged in this interdisciplinary field of data science and materials discovery for thermal applications. While algorithms for data processing and ML have become increasing sophisticated, high-quality datasets particularly suitable for thermal properties are still difficult to obtain. This is largely due to the high computational cost involved in computing various thermal properties from physical models and first-principles. Therefore, high-throughput methodology and freamework have been one of the most important approaches, which can settle the bottleneck of data deficiency. What is also challenging is our lack of understanding on the correlation of material descriptors with transport properties. ML has provided us new insights into correlations that were not physically intuitive, offering us insights into future material discoveries for thermal science. Overall, the coupling of theoretical and experimental HT techniques would become key for the development of this field, liberating us from the repetitive work of parameter sweeps and measurements towards new physics and new materials. 


\section{References}

1. T. Li, J. Song, X. Zhao, Z. Yang, G. Pastel, S. Xu, C. Jia, J. Dai, C. Chen, A. Gong, F. Jiang, Y. Yao, T. Fan, B. Yang, L. Wågberg, R. Yang and L. $\mathrm{Hu}$, Science Advances, 2018, 4, eaar3724.

2. M. Behnia, L. Maguire and G. Morrison, 5th International Conference on Thermal and Mechanical Simulation and Experiments in Microelectronics and Microsystems, 2004. EuroSimE 2004. Proceedings of the, 2004.

3. M. F. Ashby, in Materials and the Environment (Second Edition), ed. M. F. Ashby, Butterworth-Heinemann, Boston, 2013, DOI: https://doi.org/10.1016 /B978-0-12-385971-6.00009-9, pp. 227-273.

4. D. A. Broido, M. Malorny, G. Birner, N. Mingo and D. A. Stewart, Applied Physics Letters, 2007, 91, 231922.

5. S. Curtarolo, G. L. W. Hart, M. B. Nardelli, N. Mingo, S. Sanvito and O. Levy, Nature Materials, 2013, 12, 191-201.

6. G. Petretto, S. Dwaraknath, H. P. C. Miranda, D. Winston, M. Giantomassi, M. J. v. Setten, X. Gonze, K. A. Persson, G. Hautier and G.-M. Rignanese, Scientific Data, 2018, 5, 180065.

7. M. A. Blanco, E. Francisco and V. Luaña, Computer Physics Communications, 2004, 158, 57-72.

8. P. Ravindran, L. Fast, P. A. Korzhavyi, B. Johansson, J. Wills and O. Eriksson, Journal of Applied Physics, 1998, 84, 4891-4904.

9. M. de Jong, W. Chen, T. Angsten, A. Jain, R. Notestine, A. Gamst, M. Sluiter, C. Krishna Ande, S. van der Zwaag, J. J. Plata, C. Toher, S. Curtarolo, G. Ceder, K. A. Persson and M. Asta, Scientific Data, 2015, 2, 150009.

10. M. Q. Liao, Y. Liu, L. J. Min, Z. H. Lai, T. Y. Han, D. N. Yang and J. C. Zhu, Intermetallics, 2018, 101, 152-164.

11. J. A. Rogers, M. Fuchs, M. J. Banet, J. B. Hanselman, R. Logan and K. A. Nelson, Applied Physics Letters, 1997, 71, 225-227.

12. Z. Q. Wen, Y. H. Zhao, J. Z. Tian, S. Wang, Q. W. Guo and H. Hou, Journal of Materials Science, 2019, 54, 2566-2576.

13. Y. Wang, S. L. Shang, H. Fang, Z. K. Liu and L. Q. Chen, npj Computational Materials, 2016, 2, 16006.

14. H. Bao, J. Chen, X. Gu and B. Cao, ES Energy \& Environment, 2018, DOI: 10.30919/esee8c149.

15. S. A. Miller, P. Gorai, B. R. Ortiz, A. Goyal, D. Gao, S. A. Barnett, T. O. Mason, G. J. Snyder, Q. Lv, V. Stevanović and E. S. Toberer, Chem. Mater, 2017, 29, 2494-2501.

16. L. Lindsay, D. A. Broido and T. L. Reinecke, Physical Review Letters, 2013, 111, 025901.

17. D. Zhao, X. Qian, X. Gu, S. A. Jajja and R. Yang, Journal of Electronic Packaging, 2016, 138, 040802-040802-040819.

18. S. Rubin and F. F. Gettinger, Semiconductor measurement technology: Thermal resistance measurements on power transistors, 1979.

19. J. Carrete, W. Li, N. Mingo, S. Wang and S. Curtarolo, Phys. Rev. X, 2014, 4, 011019.

20. A. Seko, A. Togo, H. Hayashi, K. Tsuda, L. Chaput and I. Tanaka, Physical Review Letters, 2015, 115, 205901.

21. D. G. Cahill, S. K. Watson and R. O. Pohl, Phys. Rev. B, 1992, 46, 61316140 .

22. J. S. Kang, M. Li, H. Wu, H. Nguyen and Y. Hu, Science, 2018, DOI: 10.1126/science.aat5522, eaat5522.

23. S. Li, Q. Zheng, Y. Lv, X. Liu, X. Wang, P. Y. Huang, D. G. Cahill and B. Lv, Science, 2018, DOI: 10.1126/science.aat8982, eaat8982.

24. F. Tian, B. Song, X. Chen, N. K. Ravichandran, Y. Lv, K. Chen, S. Sullivan, J. Kim, Y. Zhou, T.-H. Liu, M. Goni, Z. Ding, J. Sun, G. A. G. U. Gamage, H. Sun, H. Ziyaee, S. Huyan, L. Deng, J. Zhou, A. J. Schmidt, S. Chen, C.W. Chu, P. Y. Huang, D. Broido, L. Shi, G. Chen and Z. Ren, Science, 2018, DOI: 10.1126/science.aat7932, eaat7932.

25. Z. Zhang, S. Hu, T. Nakayama, J. Chen and B. Li, Carbon, 2018, 139, 289298.

26. X. Xu, J. Chen, J. Zhou and B. Li, Advanced Materials, 2018, 30, 1705544.

27. W. Lee, H. Li, A. B. Wong, D. Zhang, M. Lai, Y. Yu, Q. Kong, E. Lin, J. J. Urban, J. C. Grossman and P. Yang, PNAS, 2017, 114, 8693-8697.

28. L. D. Zhao, S. H. Lo, Y. Zhang, H. Sun, G. Tan, C. Uher, C. Wolverton, V. P. Dravid and M. G. Kanatzidis, Nature, 2014, 508, 373-377.

29. C. Chiritescu, D. G. Cahill, N. Nguyen, D. Johnson, A. Bodapati, P. Keblinski and P. Zschack, Science, 2007, 315, 351-353.

30. A. Jain, G. Hautier, C. J. Moore, S. Ping Ong, C. C. Fischer, T. Mueller, K.
A. Persson and G. Ceder, Computational Materials Science, 2011, 50, 2295 2310.

31. S. Curtarolo, W. Setyawan, G. L. W. Hart, M. Jahnatek, R. V. Chepulskii, R. H. Taylor, S. Wang, J. Xue, K. Yang, O. Levy, M. J. Mehl, H. T. Stokes, D. O. Demchenko and D. Morgan, Computational Materials Science, 2012, 58, 218-226.

32. A. Jain, S. P. Ong, G. Hautier, W. Chen, W. D. Richards, S. Dacek, S. Cholia, D. Gunter, D. Skinner, G. Ceder and K. A. Persson, APL Materials, 2013, 1, 011002 .

33. S. P. Ong, W. D. Richards, A. Jain, G. Hautier, M. Kocher, S. Cholia, D. Gunter, V. L. Chevrier, K. A. Persson and G. Ceder, Computational Materials Science, 2013, 68, 314-319.

34. J. O'Mara, B. Meredig and K. Michel, JOM, 2016, 68, 2031-2034.

35. J. E. Saal, S. Kirklin, M. Aykol, B. Meredig and C. Wolverton, JOM, 2013, 65, 1501-1509.

36. K. Yang, W. Setyawan, S. Wang, M. Buongiorno Nardelli and S. Curtarolo, Nature Materials, 2012, 11, 614-619.

37. G. Ceder, MRS Bulletin, 2010, 35, 693-701.

38. R. E. Brandt, R. C. Kurchin, V. Steinmann, D. Kitchaev, C. Roat, S Levcenco, G. Ceder, T. Unold and T. Buonassisi, Joule, 2017, 1, 843-856.

39. A. Seko, T. Maekawa, K. Tsuda and I. Tanaka, Phys. Rev. B, 2014, 89, 054303 .

40. A. N. Kolmogorov, S. Shah, E. R. Margine, A. F. Bialon, T. Hammerschmidt and R. Drautz, Physical Review Letters, 2010, 105, 217003

41. A. van Roekeghem, J. Carrete, C. Oses, S. Curtarolo and N. Mingo, Phys. Rev. X, 2016, 6, 041061.

42. C. Toher, J. J. Plata, O. Levy, M. de Jong, M. Asta, M. B. Nardelli and S Curtarolo, Phys. Rev. B, 2014, 90, 174107.

43. G. Deinzer, G. Birner and D. Strauch, Phys. Rev. B, 2003, 67, 144304.

44. J. M. Skelton, S. C. Parker, A. Togo, I. Tanaka and A. Walsh, Physical Review B, 2014, 89.

45. O. Hellman and D. A. Broido, Physical Review B, 2014, 90.

46. M. S. Green, The Journal of Chemical Physics, 1954, 22, 398-413.

47. R. Kubo, Journal of the Physical Society of Japan, 1957, 12, 570-586.

48. M. Zebarjadi, K. Esfarjani, M. S. Dresselhaus, Z. F. Ren and G. Chen, Energy Environ. Sci., 2012, 5, 5147-5162.

49. P. B. Allen, Phys. Rev. B, 2013, 88, 144302.

50. J. Callaway, Physical Review, 1959, 113, 1046.

51. G. Qin and M. Hu, npj Computational Materials, 2018, 4, 3.

52. F. Legrain, J. Carrete, A. van Roekeghem, S. Curtarolo and N. Mingo, Chem. Mater, 2017, 29, 6220-6227.

53. P. Cheng and C. T. Hsu, 1999, 2, 19-38.

54. Z. Tong, M. Liu and H. Bao, International Journal of Heat and Mass Transfer, 2016, 100, 355-361.

55. M. Wang and N. Pan, International Journal of Heat and Mass Transfer, 2008, 51, 1325-1331.

56. C. Demuth, M. A. A. Mendes, S. Ray and D. Trimis, International Journal of Heat and Mass Transfer, 2014, 77, 979-994.

57. X. He, S. Chen and G. D. Doolen, Journal of Computational Physics, 1998, 146, $282-300$

58. H. Zhang and A. J. Minnich, Scientific Reports, 2015, 5, 8995.

59. H. Wei, S. Zhao, Q. Rong and H. Bao, International Journal of Heat and Mass Transfer, 2018, 127, 908-916.

60. D. B. Miracle and O. N. Senkov, Acta Materialia, 2017, 122, 448-511.

61. W. R. Zhang, P. K. Liaw and Y. Zhang, Science China-Materials, 2018, 61, $2-22$.

62. F. Y. Tian, Frontiers in Materials, 2017, 4.

63. M. Ogura, T. Fukushima, R. Zeller and P. H. Dederichs, Journal of Alloys and Compounds, 2017, 715, 454-459.

64. R. Z. Zhang, F. Gucci, H. Y. Zhu, K. Chen and M. J. Reece, Inorganic Chemistry, 2018, 57, 13027-13033.

65. H. Q. Song, F. Y. Tian and D. P. Wang, Journal of Alloys and Compounds, 2016, 682, 773-777.

66. S. Wang, T. Zhang, H. Hou and Y. H. Zhao, Physica Status Solidi B-Basic Solid State Physics, 2018, 255.

67. T. T. Zuo, M. C. Gao, L. Z. Ouyang, X. Yang, Y. Q. Cheng, R. Feng, S. Y. Chen, P. K. Liaw, J. A. Hawk and Y. Zhang, Acta Materialia, 2017, 130, 1018. 
68. F. Kormann, Y. Ikeda, B. Grabowski and M. H. F. Sluiter, npj Comput. Mater, 2017, 3, 9.

69. H. Babaei, P. Keblinski and J. M. Khodadadi, Journal of Applied Physics, 2012, 112.

70. A. Giri, J. L. Braun, C. M. Rost and P. E. Hopkins, Scripta Materialia, 2017, 138, 134-138.

71. S. W. Kao, J. W. Yeh and T. S. Chin, Journal of Physics-Condensed Matter, 2008, 20.

72. M. Caro, L. K. Beland, G. D. Samolyuk, R. E. Stoller and A. Caro, Journal of Alloys and Compounds, 2015, 648, 408-413.

73. A. Giri, J. L. Braun and P. E. Hopkins, Journal of Applied Physics, 2018, 123.

74. S. N. H. Eliassen, A. Katre, G. K. H. Madsen, C. Persson, O. M. Lovvik and K. Berland, Physical Review B, 2017, 95.

75. M. Arrigoni, J. Carrete, N. Mingo and G. K. H. Madsen, Physical Review B,
2018, 98, 8.

76. in Collected Papers of P.L. Kapitza, ed. D. Ter Haar, Pergamon, 1965, DOI https://doi.org/10.1016/B978-0-08-010973-2.50008-2, pp. 581-624.

77. W. A. Little, Canadian Journal of Physics, 1959, 37, 334-349.

78. E. T. Swartz and R. O. Pohl, Reviews of Modern Physics, 1989, 61, 605-668.

79. E. S. Landry and A. J. H. McGaughey, Phys. Rev. B, 2009, 80, 165304.

80. Z. Liang and M. Hu, Journal of Applied Physics, 2018, 123, 191101.

81. T. Zhan, S. Minamoto, Y. Xu, Y. Tanaka and Y. Kagawa, AIP Advances, 2015, 5, 047102

82. N. Yang, T. Luo, K. Esfarjani, A. Henry, Z. Tian, J. Shiomi, Y. Chalopin, B. $\mathrm{Li}$ and G. Chen, Journal of Computational and Theoretical Nanoscience, 2015, 12, 168-174.

83. K. Gordiz and A. Henry, eprint arXiv:1407.6410, 2014, arXiv:1407.6410.

84. Y. Zhang, D. Ma, Y. Zang, X. Wang and N. Yang, Frontiers in Energy Research, 2018, 6, 48 . 